\title{
What shapes amino acid and sugar composition in Mediterranean floral nectars?
}

\author{
Theodora Petanidou, André Van Laere, Willem N. Ellis and Erik Smets
}

Petanidou, T., Van Laere, A., Ellis, W. N. and Smets, E. 2006. What shapes amino acid and sugar composition in Mediterranean floral nectars? - Oikos 115: 155-169.

We studied the amino acid (AA) composition of the floral nectars of 73 plant species occurring in a phryganic (East Mediterranean garrigue) community and investigated whether AA and sugar composition is shaped by evolutionary (plant phylogeny), ecological (flowering time as a direct effect of summer drought) and coevolutionary (pollinator partnership) constraints. Our study utilised an extensive plant-pollinator matrix compiled in the same area where the plants had been sampled.

Using HPLC we detected 22 AA compounds/groups of compounds, out of which 15 were commonly present in almost all nectars. Among all AAs, phenylalanine was the most abundant, especially in keystone ("cornucopian") plant species visited by many insect species, such as the majority of the Lamiaceae. Amino acid quantities were transformed into percentages ( $\%$ of each AA over the total AA content of a flower). Sugar composition was similarly expressed as \% of each of the three sugars (glucose, fructose, sucrose) over the total content of these sugars; a number of other sugars, occurring in only a few plant species and in very low quantities were disregarded. The number of insect species of a particular family or guild was taken as a measure of the attraction of a nectar compound for such a family (guild).

We found that taxonomical plant group had a weakly significant effect on nectar composition while neither life form nor flowering season had a discernable effect. Pollinators' preference had the most important effect, with phenylalanine being the most consequent discriminatory compound for the response of the nectar consumers in phrygana, predominantly for long tongued bees, especially for Megachilidae. Gamma-aminobutyric acid (GABA) had a similar, even stronger influence on bees (long tongued bees, Anthophoridae, Andrenidae) and flies (Syrphidae and other Diptera), whereas asparagine behaved as a general repellent together with tryptophane (rather as repellent). Considering total sugar and AA contents, as well as the volume of nectar, we found that total AA content was positively related to the number of species of long tongued bees and included families visiting the phryganic species; nectar volume was negatively related to flies (both hover flies and remaining Diptera), whereas total sugar content was not significant for any guild. We argue that due to the highly concentrated nectars in the dry Mediterranean communities that are characterised by outstanding melittophily, sugars play a less important role as phagostimulants compared to AAs in floral nectars. This is why phenylalanine, a phagostimulant tested earlier on honeybees, appears to be of high importance in phrygana, especially with long tongued bees and Megachilids as the main selective agents for phenylalanine-rich nectars. The role of GABA, a strongly $\mathrm{NaCl}$-dependent $\mathrm{AA}$, may be similar, probably because of the associated presence of $\mathrm{NaCl}$.

T. Petanidou, Laboratory of Biogeography and Ecology, Dept of Geography, Univ. of the Aegean, University Hill, GR-81100 Mytilene, Greece (t.petanidou@aegean.gr). - A. Van Laere, Laboratory of Molecular Plant Physiology, Inst. of Botany and Microbiology, K.U. Leuven, Kasteelpark Arenberg 31, BE-3001 Leuven, Belgium. - W. N. Ellis, Zoological Museum, Section of Entomology, Univ. of Amsterdam, Plantage Middenlaan 64, NL-1018 DH Amsterdam, the Netherlands. - Erik Smets, Laboratory of Systematics, Inst. of Botany and Microbiology, Kasteelpark Arenberg 31, K.U. Leuven, BE-3001 Leuven, Belgium.

Accepted 10 April 2006

Subject Editor: Jane Memmott

Copyright (C) OIKOS 2006

ISSN 0030-1299 
Functioning almost exclusively as floral reward to pollinators, nectar compostition is likely to be subject to selection pressures imposed by the nectar feeders. As a consequence, and due to "convergent coevolution", nectar characteristics tend to be similar for plants exhibiting the same pollination syndrome and different between closely related plants with different pollinators (Pyke and Waser 1981, Baker and Baker 1982). At the same time, nectar traits are subject to influence by phylogenetic affiliations and ecological factors imposed by the habitat (Corbet 1990, Petanidou and Smets 1996, Petanidou et al. 1999, 2000, Petanidou 2005).

Most of the nectar literature focuses on sugars, the major components of floral nectars. The amounts and sugar concentration of nectar have been related to pollinator type (Percival 1961, 1965, Baker and Baker 1975), especially considering the sucrose/hexose ratio (Wykes 1952, Percival 1961, Baker and Baker 1979, 1982, 1983, 1990, Southwick 1982, Stiles and Freeman 1993, Petanidou et al. 1996, 2000, Petanidou 2005). Amino acids (AAs) in nectars, and their evolutionary significance, have also received attention (Baker and Baker 1973a, 1973b, 1982, 1986, Baker et al. 1978, Gottsberger et al. 1984, 1989, 1990, Pais et al. 1986, Erhardt 1991, 1992, Petanidou et al. 1996, 2000). In a long series of studies based on the analysis of ca 1500 species, Baker and Baker (1973a, 1973b, 1982, 1986) proposed that the amount of AAs present in nectars are directly in connection with their pollination systems. These conclusions were disputed by Gottsberger et al. $(1984,1989)$, but the debate has never been concluded on the basis of hard evidence from a large scale study. Today the "consensus view" is that plants adapted to pollination by butterflies have high concentrations of AAs compared to plants that are bird or bee pollinated (Gardener and Gillman 2002).

Dafni and Kevan (1994) proposed that AAs in nectar may have a positive effect in attracting pollinators. When reviewing the recent literature on AA preference of flower-visiting insects, Gardener and Gillman (2002) also concluded that not only honeybees but also flies show a preferential response to AAs contained in nectarmimicking solutions. The authors emphasize the contribution of AAs to the taste of nectar (rather than the nutritive value), an aspect that has been neglected by the nectar literature. This is related to their finding that the amounts of AAs in nectars are greatly variable in nature whereas their composition (i.e. \% contribution of the particular AAs) is much less variable (Gardener and Gillman 2001). This implies that low AA quantities may act mainly as attractants or phagostimulants in the natural floral nectars found in nature. In this respect, the phagostimulatory effect of phenylalanine which was found to alter the response of honeybees among several single AAs tested, appears to be very significant (Inouye and Waller 1984).

In this study we investigate the factors shaping the AA and sugar (AA\&S) composition in floral nectars. Because of the type and diversity of the factors explored, it was important that our approach should be carried out at a community level and encompass an entire plantpollinator community. The study investigates the pollinator preference for nectar AA\&S as recorded in nature at the community level and within the actual plantpollinator (p-p) interaction network. The novelty of this study in comparison to earlier ones is that it explores:

1) AA\&S nectar composition in a number of native plant species being under the same ecological constraints, and

2) the preference for AA\&S nectar composition by bees and other insects visiting the plants at the community level, considering their taxonomy and guild membership on the basis of all plantpollinator interactions recorded.

The study was conducted in a phrygana, a typical low shrub occupying the driest parts of the precipitation gradient in the East Mediterranean, comparable to the garrigue in the West Mediterranean. Its physiognomy is determined by seasonally dimorphic woody plants, such as Thymus capitatus, Sarcopoterium spinosum, and Satureja thymbra, but in terms of species diversity, annual plants are the predominant life form (Petanidou and Vokou 1993, Petanidou et al. 1995). Despite the first impression of precariousness and poverty, phrygana bears a rich flora, and many nesting possibilities for ground and twig nesting bees (Petanidou and Ellis 1993, Potts et al. 2004). This high diversity is maintained despite of strong environmental pressures, in the first place the prolonged summer drought of the Mediterranean climate, exacerbated by grazing and fires. In fact, the Mediterranean basin, and especially its low scrublands, has been considered as one of the world's centres of bee speciation (Michener 1979, 2000) supporting some of the most diverse plant-pollinator communities (Petanidou 1991, Petanidou and Ellis 1993, 1996, Petanidou et al. 1995, Petanidou and Potts 2006). The fact that background data is available on flowering and on p-p food web at the community level, together with the high insect (especially bee) diversity of phrygana, and the fact that ecological constraints play a significant role in shaping many pollination and nectar attributes in the community (Petanidou et al. 1995, 2000), make phrygana an excellent research habitat.

Considering that Mediterranean communities are strongly influenced by ecological limitations that may be more influential than phylogenetic ones (Petanidou et al. 1995), we ask whether AA\&S composition is 
influenced by phylogenetic or/and ecological constraints (life form, time of flowering) or/and whether it is a result of coevolution with floral nectar consumers. The study was carried out on the largest possible number of entomophilous plant species within the community studied. Yet, because of the consistency in their ecological origin (all were typical phryganic plant species, no cultivated or invading species) we assume that all species have undergone similar constraints, which allows us to draw conclusions concerning whether AA\&S composition is shaped by phylogenetic, ecological or coevolutionary constraints.

Our predictions are as follows: if AA\&S composition is shaped by phylogenetic constraints, this must be reflected in differences of AA\&S composition among high level plant taxonomical groups. If ecological constraints shape AA\&S composition, this composition is expected to differ among life forms or/and to depend on flowering season. Finally, if AA\&S composition is governed by coevolutionary constraints (i.e. pollinators), then plants will differ in AA\&S composition according to the insect guilds and families they is pollinated by (Petanidou et al. 1995, Petanidou 2005).

In a separate study Petanidou (2005) explored the driving forces shaping the sugar composition alone within the floral nectars of the phryganic plants in the same community. She found that both ecological and evolutionary attributes are significant in shaping sugar composition in the nectars of the same phryganic plants. In particular she found that sugar composition in the nectars, measured as sucrose/hexose ratio, is associated with flowering season (ecological constraint), plant family membership (evolutionary constraints), as well as with particular pollinator guilds, with the bee family Megachilidae constituting a major selective factor for "high sucrose" nectars.

\section{Material and methods}

\section{Study site and species}

The study was conducted in a phryganic community within the nature reserve "I. and A. Diomedes Botanical Garden of Athens University". Detailed description of the site is available in earlier studies (Petanidou and Ellis 1993, 1996, Petanidou et al. 1995).

All major nectar producing species (73) occurring in the reserve were considered in the study, comprising ca $65 \%$ of the local nectariferous flora (Petanidou et al. 1995, Petanidou and Smets 1995, Table 1).

\section{Nectar measurements and analyses}

Nectar measurements and collection were carried out during 1992 and 1993 and, in very few cases, in 1994 during the flowering peak of each species (Petanidou et al. 1995, Petanidou and Smets 1995). Because AA quantity increases with senescence, we used flowers at their first day of anthesis (Petanidou et al. 1996).

All nectars analyzed in this study were collected at the same time and from the same plants used to measure nectar volume (Petanidou and Smets 1995) and to analyse for nectar sugars (Petanidou 2005). Laboratory AA chemical analyses were conducted on the same nectars used to carry out sugar analyses. This is why nectar volume and sugar data used in this study originate from the above two studies, respectively.

Flowers were selected at random and covered in bud stage with bridal veil in the eve of the collection day to prevent nectar removal and contamination by insects. Nectar was collected towards noon to early afternoon (11:00-14:00 h) except for the nocturnally flowering species Capparis spinosa sampled between 9:30 and 10:00 h. The nectar of each flower was picked up directly on a Whatman No. 1 small paper wick prepared in advance. During the collection, particular care was taken to avoid any contamination of the nectar by pollen or other structural plant tissue. After nectar collection the paper wicks were fixed on stainless steel pins that had been carefully cleaned with acetone, then placed on styrofoam blocks and left to air-dry. Finally they were stored at $\pm 5^{\circ} \mathrm{C}$ in air-tight containers over silica gel until analysis. Touching with the fingers or other possible contaminating sources was carefully avoided (Petanidou et al. 1996). Number of flowers analyzed per plant species varied between 1 and 51 .

AA analysis of nectar samples was carried out by means of high pressure liquid chromatography (HPLC). Before analysis, nectar content of each wick was dissolved in $1 \mathrm{ml}$ of distilled water in a microcentrifuge tube by intermittent vortexing at room temperature for at least one hour. Finally, the tubes were centrifuged to remove paper particles (Petanidou et al. 1996).

For chemical analysis, $100 \mu \mathrm{l}$ of the diluted nectar were treated for $3 \mathrm{~min}$ with $50 \mu \mathrm{l}$ of $0.02(\mathrm{w} / \mathrm{v})$ ophthaldialdehyde and $0.02(\mathrm{v} / \mathrm{v})$ 2-mercaptoethanol in $0.1 \mathrm{M} \mathrm{NaHCO}_{3}, \mathrm{pH} 9.5$, immediately before injection. Twenty $\mu \mathrm{l}$ of the AA derivatives were then injected on a $200 \times 3$ mm ChromSpher C8 (Chrompack, Middelburg, the Netherlands) HPLC-column. Elution of the column occurred at a constant flow rate of $0.8 \mathrm{ml} \mathrm{min}^{-1}$ using a linear gradient from 20 to $100 \%(\mathrm{v} / \mathrm{v})$ of solvent B over $15 \mathrm{~min}$ and continuing with solvent $\mathrm{B}$ for $10 \mathrm{~min}$. Elution solvents were: $\mathrm{A}=$ methanol $/ \mathrm{H}_{2} \mathrm{O} /$ tetrahydrofuran (2/96/2) containing $50 \mathrm{mM} \mathrm{Na} \mathrm{HPO}_{4}$ and 50 $\mathrm{mM}$ sodium acetate (neutralized to $\mathrm{pH} 7.5$ with acetic acid), and $\mathrm{B}=$ methanol $/ \mathrm{H}_{2} \mathrm{O}(65 / 35 \mathrm{v} / \mathrm{v}$ ) (Petanidou et al. 1996). The derivatives were fluorometrically detected (1700 Fluorescence Monitor, Bio Rad, USA; 
Table 1. The plant species of phrygana studied for their nectar amino acids and sugars using HPLC analysis.

\begin{tabular}{|c|c|c|c|c|c|}
\hline $\begin{array}{l}\text { Date of nectar } \\
\text { collection }\end{array}$ & Plant species $^{1}$ & Abbreviation & Life form ${ }^{2,3}$ & $\begin{array}{c}\text { Nectar }^{3} \\
\text { volume }^{3} \\
\mu 1 \text { flower }^{-1}\end{array}$ & $\begin{array}{l}\text { Midpoint of } \\
\text { flowering } \\
\text { calendar day }\end{array}$ \\
\hline
\end{tabular}

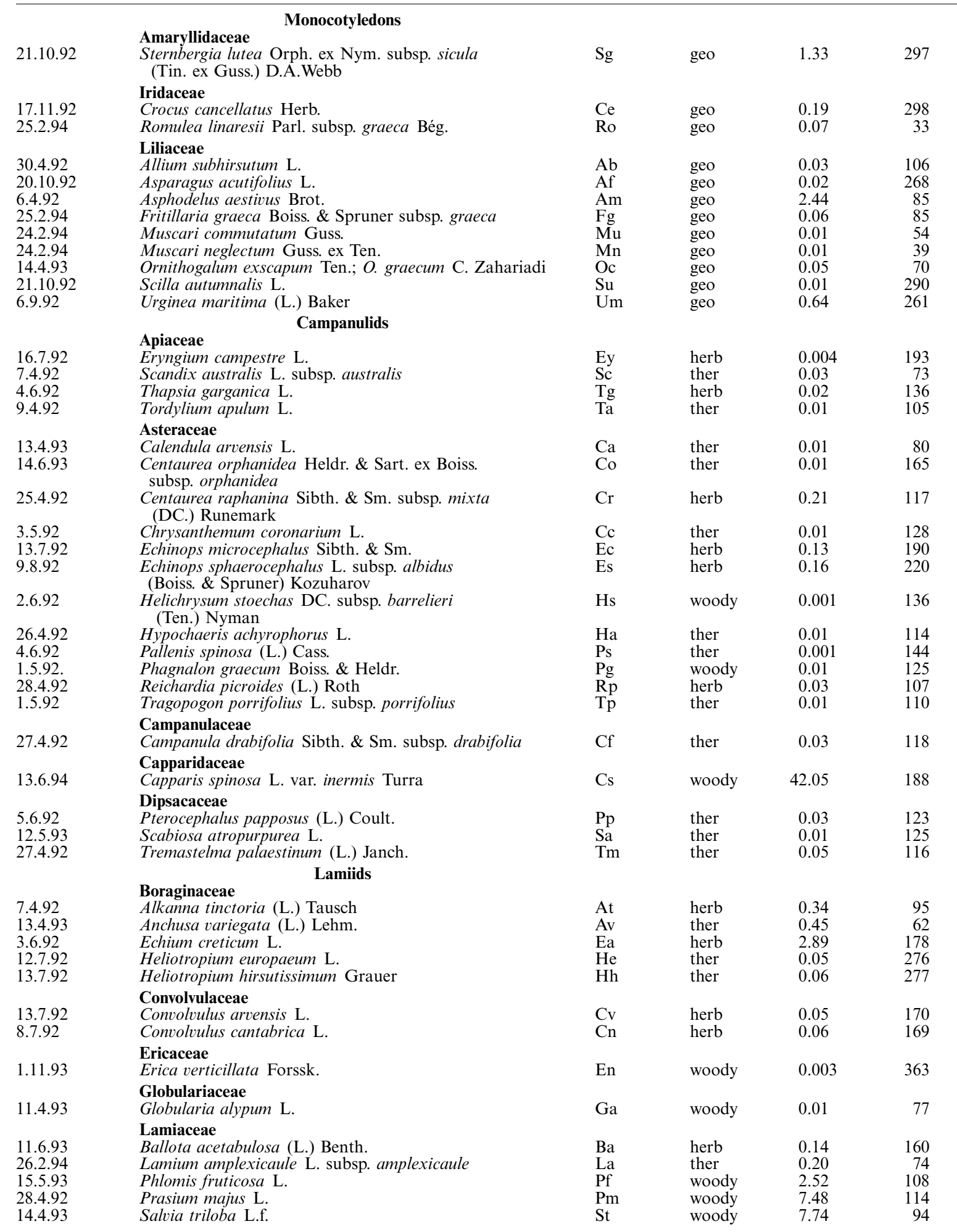


Table 1 (continued)

\begin{tabular}{|c|c|c|c|c|c|}
\hline $\begin{array}{l}\text { Date of nectar } \\
\text { collection }\end{array}$ & Plant species ${ }^{1}$ & Abbreviation & Life form ${ }^{2,3}$ & $\begin{array}{l}\text { Nectar } \\
\text { volume }^{3} \\
\mu 1 \text { flower }\end{array}$ & $\begin{array}{l}\text { Midpoint of } \\
\text { flowering } \\
\text { calendar day }\end{array}$ \\
\hline $\begin{array}{l}9.4 .92 \\
16.5 .93 \\
2.6 .92 \\
5.6 .92 \\
4.6 .92 \\
8.7 .92\end{array}$ & $\begin{array}{l}\text { Salvia verbenaca } \mathrm{L} \text {. } \\
\text { Satureja thymbra L. } \\
\text { Stachys cretica } \text { L. subsp. cretica } \\
\text { Teucrium chamaedrys L. } \\
\text { Teucrium polium L. subsp. capitatum (L.) Arcang. } \\
\text { Thymus capitatus (L.) Hoffmanns. \& Link } \\
\text { Malvids }\end{array}$ & $\begin{array}{l}\mathrm{Sb} \\
\mathrm{Sj} \\
\mathrm{Sy} \\
\mathrm{Td} \\
\mathrm{Te} \\
\mathrm{Tc}\end{array}$ & $\begin{array}{l}\text { ther } \\
\text { woody } \\
\text { herb } \\
\text { woody } \\
\text { woody } \\
\text { woody }\end{array}$ & $\begin{array}{l}0.33 \\
0.05 \\
0.59 \\
0.50 \\
0.06 \\
0.10\end{array}$ & $\begin{array}{r}85 \\
137 \\
137 \\
135 \\
156 \\
171\end{array}$ \\
\hline $\begin{array}{l}19.4 .93 \\
2.5 .92\end{array}$ & $\begin{array}{l}\text { Brassicaceae } \\
\text { Eruca vesicaria Cav. subsp. sativa (Mill.) Thell. } \\
\text { Sisymbrium orientale L. } \\
\text { Cistaceae }\end{array}$ & $\begin{array}{l}\text { Ev } \\
\text { So }\end{array}$ & $\begin{array}{l}\text { ther } \\
\text { ther }\end{array}$ & $\begin{array}{l}0.13 \\
0.01\end{array}$ & $\begin{array}{l}113 \\
119\end{array}$ \\
\hline $\begin{array}{l}30.4 .92 \\
2.5 .92\end{array}$ & $\begin{array}{l}\text { Cistus parviflorus Lam. } \\
\text { Cistus salvifolius L. } \\
\text { Malvaceae }\end{array}$ & $\begin{array}{l}\text { Cp } \\
\text { Ci }\end{array}$ & $\begin{array}{l}\text { woody } \\
\text { woody }\end{array}$ & $\begin{array}{l}0.05 \\
0.02\end{array}$ & $\begin{array}{l}125 \\
104\end{array}$ \\
\hline 5.6 .92 & $\begin{array}{l}\text { Alcea pallida (Willd.) Waldst. \& Kit. } \\
\text { Resedaceae }\end{array}$ & Ap & herb & 2.54 & 160 \\
\hline 27.4 .92 & $\begin{array}{l}\text { Reseda alba } \mathrm{L} \\
\text { Rutaceae }\end{array}$ & $\operatorname{Re}$ & herb & 0.10 & 104 \\
\hline 5.6 .92 & $\begin{array}{l}\text { Ruta graveolens L. } \\
\text { Thymelaeaceae }\end{array}$ & $\mathrm{Rg}$ & herb & 0.32 & 145 \\
\hline 27.2 .93 & $\begin{array}{l}\text { Thymelaea hirsuta (L.) Endl. } \\
\text { Lower dicotyledons } \\
\text { Caryophylaceae }\end{array}$ & $\mathrm{Th}$ & woody & 0.000 & 25 \\
\hline $\begin{array}{l}25.4 .92 \\
9.4 .92\end{array}$ & $\begin{array}{l}\text { Petrorhagia velutina (Guss.) P.W.Ball \& Heywood } \\
\text { Silene colorata Poir. }\end{array}$ & $\begin{array}{l}\mathrm{Pv} \\
\mathrm{Si}\end{array}$ & $\begin{array}{l}\text { ther } \\
\text { ther }\end{array}$ & $\begin{array}{l}0.04 \\
0.06\end{array}$ & $\begin{array}{r}106 \\
95\end{array}$ \\
\hline $\begin{array}{l}10.7 .92 \\
5.6 .92 \\
13.4 .92\end{array}$ & $\begin{array}{l}\text { Ranunculaceae } \\
\text { Delphinium peregrinum L. } \\
\text { Nigella arvensis L. } \\
\text { Ranunculus sprunerianus Boiss. } \\
\quad \text { Fabids }\end{array}$ & $\begin{array}{l}\mathrm{Dp} \\
\mathrm{Ng} \\
\mathrm{Ra}\end{array}$ & $\begin{array}{l}\text { ther } \\
\text { ther } \\
\text { geo }\end{array}$ & $\begin{array}{l}0.52 \\
0.30 \\
0.08\end{array}$ & $\begin{array}{r}185 \\
169 \\
98\end{array}$ \\
\hline 15.7 .92 & $\begin{array}{l}\text { Cucurbitaceae } \\
\text { Ecballium elaterium (L.) A. Rich. } \\
\text { Euphorbiaceae }\end{array}$ & $\mathrm{Ee}$ & herb & 0.03 & 214 \\
\hline 11.4 .93 & $\begin{array}{l}\text { Euphorbia acanthothamnos Heldr. \& Sart. ex Boiss. } \\
\text { Fabaceae }\end{array}$ & $\mathrm{Eu}$ & woody & 0.25 & 79 \\
\hline $\begin{array}{l}16.5 .93 \\
8.4 .92 \\
17.4 .93 \\
29.4 .92 \\
8.4 .92\end{array}$ & $\begin{array}{l}\text { Anthyllis hermanniae } \mathrm{L} . \\
\text { Astragalus monspessulanus } \mathrm{L} . \\
\text { Hymenocarpos circinnatus } \text { (L.) Savi } \\
\text { Psoralea bituminosa L. } \\
\text { Trifolium stellatum L. }\end{array}$ & $\begin{array}{l}\text { Ah } \\
\text { Ao } \\
\text { Hc } \\
\text { Pso } \\
\text { Ts }\end{array}$ & $\begin{array}{l}\text { woody } \\
\text { herb } \\
\text { ther } \\
\text { herb } \\
\text { ther }\end{array}$ & $\begin{array}{l}0.01 \\
0.28 \\
0.01 \\
0.24 \\
0.04\end{array}$ & $\begin{array}{r}132 \\
102 \\
96 \\
135 \\
98\end{array}$ \\
\hline
\end{tabular}

${ }^{1}$ Nomenclature used is according to "The International Plant Names Index" (2004)

${ }^{2}$ Life forms are: geophytes (geo), therophytes or annuals (ther), herbaceous perennials (herb), and woody perennials (woody)

3 from Petanidou et al. (1995) and Petanidou and Smets (1995)

excitation at $350 \mathrm{~nm}$, emission at $440 \mathrm{~nm}$ ), and quantified by automatic integration after calibration of the system with known AA quantities. Although proline is known to be important to pollinators, it could not be identified by this method; the same held for cysteine (same as in Petanidou et al. 1996, 2000). Because the pairs glutamine and histidine, glycine and threonine, as well as alanine and tyrosine, could not be well separated in many plant species, these AAs will be referred to as AA pairs in this paper.

\section{Plant flowering time, life form and phylogeny}

In order to investigate the importance of flowering time during the year in shaping nectar AA\&S composition, we determined the midpoint of flowering time of the plant species studied. Species having their midpoint in calendar days 61-151 were assigned as spring-, those between 152-240 as summer-, between $241-320$ as autumn- and between 321-60 as winter-flowering (Petanidou et al. 1995). The life form types are described in Petanidou et al. (1995).

Conceivably, the AA\&S values might be determined by the phylogeny of the plants, rather than by ecological constraints. To test this we grouped the plant species into a limited number of supra-ordinal categories, as defined by A.P.G. (1998) and Soltis et al. (2005). These groups, in "rising" taxonomical order, are: monocots, lower dicots, fabids, malvids, lamiids and campanulids. 


\section{Flower visitors and plants used in the analyses}

In order to detect whether pollinator species had a differential response to the AA\&S composition of flower nectars, we used the community data matrix from Petanidou (1991) that was also used as a basis in similar studies (Petanidou and Ellis 1996, Petanidou 2005, Petanidou and Potts 2006). The data were collected from March 1983 to May 1987 from all entomophilous plant species of the same community where the nectars were collected. As "pollinators", all flower visitors were considered if visiting the flowers repeatedly, irrespectively of their "quality" (pollinator effectiveness), as normally done in such community studies (Waser and Ollerton 2006). The matrix contains the plant-pollinator interactions for 70 out of the 73 plant species analyzed here (no data for the species Echinops sphaerocephalus subsp. albidus, Teucrium chamaedrys and Romulea linaresii, which were excluded from the plant-pollinator interaction analyses). In addition, another three plant species had to be excluded from all the data analyses because either their nectar value was taken from the chemical analysis of one flower only (Fritillaria graeca), or because there was a high risk of nectar contamination (Thymelaea hirsuta and Crocus cancellatus). Therefore, our analysis is based on 67 plant species visited by 565 insect species, resulting in 1865 plant-pollinator interactions which were basically considered in the calculations.

For each plant species, we counted the number of species visiting them, grouped into guilds and into major bee families. We recognized the following guilds: Coleoptera, Hemiptera, Lepidoptera. Syrphidae, other Diptera, Apidae, long tongued (solitary) bees (viz. Anthophoridae and Megachilidae), short tongued bees (viz. Andrenidae, Halictidae and Colletidae; the single observation of a melittid species was disregarded), and wasps (i.e. other Hymenoptera). The numbers so obtained (referred to below as "visitors per guild" or "visitors per family") were taken as indicative of the importance of a plant for a guild or family.

\section{Data analysis}

The AA values from all laboratory analyses were calculated as amounts of AA per flower ( = AA content). This was the average of several separate runs (mainly 311; Table 2), each one on the basis of one flower where nectar secretion was sufficient (e.g. in Lamiaceae). In cases where nectar secretion per flower was too low, due to the little nectar secreted by a flower (Petanidou and Smets 1995), the nectar samples were pooled for HPLC analysis (Table 2). Information about chemical and data analysis for sugars is given in Petanidou (2005).
In all statistical analyses we used the \% composition of a particular AA (i.e. proportion of a single AA over the total AA content in the nectar of a plant species; in pmoles) instead of using absolute AA concentrations. Similarly, values of glucose, fructose and sucrose (in nmoles) were transformed into percentages over the total sugar content. The use of proportions reduces problems of concentration changes due to evaporation and interflower variability of the total AA content, therefore it may account for more reliable results both in chemical and biological aspect (Lanza et al. 1995, Gardener and Gillman 2001).

In order to investigate the effects of ecological or phylogenetic factors on nectar composition, avoiding at the same time any possible autocorrelations within our data set, we used a multifactorial approach (multivariate ANOVA, stepwise multiple regressions, PCA). In the analyses we considered volume, all AAs, and only the three major sugars (viz. sucrose, glucose, fructose, i.e. discarding minor sugars found in traces) of the nectars.

For statistical analysis, all nectar values (viz. AA and sugar percentages, nectar volumes) and numbers of visitors were effectively normalized by transforming them to their $\ln$ values $\left(x^{\prime}=\ln (x+1)\right)$. Statistical analyses were done using the Data Desk package (Velleman 2005).

Whenever used in the text, values are followed by their SE. SE values are omitted in Table 2 in order to avoid confusion (SE would give the true flower-to-flower variability only in cases where nectar was analysed per flower, as it was in the case of the family Lamiaceae; Petanidou et al. 2000).

\section{Results}

In Table 1 we have listed all of the 73 plant species studied in the community. Twenty-two AA compounds or groups of AA compounds were found in their floral nectars (Table 2). Out of these, 19 were identifiable on the basis of the AA references used in the HPLC analysis. The remaining three non-identified AAs are summed up and listed under "unknown" in Table 2.

Phenylalanine was the most abundant AA in the nectars (mean value $0.72 \pm 0.233$ nmoles flower ${ }^{-1}, \mathrm{n}=$ 70 , Table 2). It also had the the highest proportion in the nectars over all plant species studied (\% of phenylalanine: $17.1 \pm 2.65, \mathrm{n}=70$ ). Within the whole community, 21 plant species were phenylalanine-rich (having nectars with $>30 \%$ phenylalanine), among them all the Lamiaceae $(47.2 \%$ in average, including Lamium amplexicaule having a very low value). Among the most prominent phenylalanine-rich species were Stachys cretica $(80 \%)$, Phlomis fruticosa $(55 \%)$, Satureja thymbra $(48 \%)$, Urginea maritima (73\%), Asphodelus aestivus (46\%) and Thapsia garganica $(46 \%)$, all keystone species within 


\begin{tabular}{|c|c|c|c|c|c|c|c|c|c|c|c|c|c|c|c|c|c|c|c|c|c|c|c|c|c|c|}
\hline \multirow{3}{*}{$\begin{array}{l}\text { Plant } \\
\text { species }^{1}\end{array}$} & \multirow{3}{*}{$\begin{array}{c}\mathrm{N} \\
\left(\mathrm{N}^{\prime}\right)^{2}\end{array}$} & \multicolumn{21}{|c|}{ Amino acids } & \multicolumn{4}{|c|}{ Sugars ${ }^{6}$} \\
\hline & & $\begin{array}{l}\text { Total } \\
\mathrm{AAs}^{3}\end{array}$ & $\mathrm{Asp}^{4}$ & Glu & Asn & Ser & $\begin{array}{l}\mathrm{His}+ \\
\mathrm{Gln}^{5}\end{array}$ & $\begin{array}{c}\text { Gly }+ \\
\text { Thr }^{5}\end{array}$ & $\operatorname{Arg}$ & $\begin{array}{l}\text { Tyr }+ \\
\mathrm{Ala}^{5}\end{array}$ & Gaba & Trp & Met & Val & Phe & Ile & Leu & Orn & Lys & H-Ser & Unknown & $\beta$-Ala & $\begin{array}{c}\text { Total } \\
\text { sugars }^{3}\end{array}$ & Glucose & Fructose & Sucros \\
\hline & & \multicolumn{21}{|c|}{ pmoles/flower } & \multicolumn{4}{|c|}{ nmoles/flower } \\
\hline $\mathrm{Ab}$ & $10(3)$ & 869 & 49 & 50 & 14 & 67 & 191 & 80 & 16 & 121 & 0 & 63 & 9 & 66 & 30 & 3 & 22 & 47 & 4 & 0 & 39 & 0 & 300 & 122 & 125 & 53 \\
\hline Af & 55 (6) & 583 & 16 & 21 & 54 & 91 & 108 & 54 & 5 & 38 & 3 & 16 & 3 & 25 & 17 & 7 & 9 & 3 & 12 & 0 & 16 & 84 & 222 & 93 & 127 & 1 \\
\hline $\mathrm{Ah}$ & $11(4)$ & 240 & 7 & 4 & 3 & 19 & 15 & 40 & 3 & 15 & 0 & 11 & 0 & 94 & 0 & 4 & 25 & 0 & 0 & 0 & 0 & 0 & 42 & 0 & 0 & 41 \\
\hline $\mathrm{Am}^{*}$ & $3(3)$ & 8880 & 90 & 50 & 62 & 600 & 534 & 472 & 82 & 293 & 1291 & 50 & 25 & 142 & 4097 & 77 & 90 & 61 & 69 & 0 & 795 & 0 & 21326 & 7845 & 8035 & 5447 \\
\hline Ao & $13(6)$ & 1621 & 29 & 19 & 263 & 128 & 23 & 119 & 15 & 26 & 94 & 13 & 28 & 74 & 6 & 14 & 19 & 26 & 24 & 150 & 552 & 0 & 1188 & 265 & 25 & 898 \\
\hline Ap* & $3(3)$ & 5758 & 280 & 154 & 574 & 195 & 445 & 473 & 237 & 1610 & 0 & 75 & 0 & 287 & 9 & 17 & 50 & 140 & 82 & 0 & 1129 & 0 & 22637 & 10456 & 11351 & 831 \\
\hline At & $6(4)$ & 456 & 18 & 7 & 11 & 32 & 7 & 53 & 5 & 173 & 36 & 9 & 0 & 52 & 39 & 0 & 12 & 0 & 0 & 0 & 0 & 0 & 1136 & 421 & 148 & 567 \\
\hline $\mathrm{Av}$ & $13(5)$ & 2030 & 54 & 75 & 1285 & 47 & 76 & 101 & 76 & 75 & 14 & 84 & 0 & 26 & 40 & 6 & 13 & 22 & 9 & 0 & 27 & 0 & 731 & 216 & 130 & 385 \\
\hline $\mathrm{Ba}^{*}$ & $10(10)$ & 2620 & 70 & 30 & 0 & 370 & 160 & 430 & 0 & 250 & 0 & 0 & 30 & 100 & 920 & 20 & 50 & 140 & 50 & 0 & 0 & 0 & 338 & 47 & 12 & 279 \\
\hline $\mathrm{Ca}$ & $12(3)$ & 1336 & 16 & 14 & 33 & 56 & 316 & 69 & 20 & 46 & 46 & 46 & 0 & 151 & 262 & 57 & 51 & 32 & 109 & 0 & 10 & 0 & 106 & 50 & 52 & 3 \\
\hline $\mathrm{Cc}$ & $24(5)$ & 436 & 20 & 9 & 4 & 24 & 17 & 43 & 18 & 131 & 0 & 12 & 0 & 7 & 56 & 3 & 7 & 29 & 14 & 0 & 43 & 0 & 111 & 58 & 50 & 4 \\
\hline $\mathrm{Ce}$ & $13(3)$ & 18033 & 597 & 442 & 707 & 1610 & 2866 & 7000 & 138 & 2429 & 0 & 167 & 170 & 737 & 232 & 242 & 230 & 249 & 55 & 0 & 162 & 0 & 537 & 219 & 210 & 109 \\
\hline $\mathrm{Cf}$ & $6(3)$ & 1271 & 25 & 24 & 13 & 51 & 23 & 85 & 26 & 148 & 0 & 109 & 0 & 0 & 534 & 15 & 31 & 62 & 53 & 0 & 72 & 0 & 1195 & 308 & 358 & 529 \\
\hline $\mathrm{Ci}$ & $10(3)$ & 9663 & 1518 & 781 & 285 & 630 & 1569 & 539 & 578 & 1007 & 212 & 410 & 24 & 437 & 324 & 276 & 264 & 475 & 237 & 0 & 96 & 0 & 3648 & 1442 & 1627 & 579 \\
\hline $\mathrm{Cn}$ & $10(4)$ & 1080 & 23 & 26 & 38 & 41 & 57 & 66 & 17 & 76 & 35 & 38 & 13 & 27 & 546 & 8 & 20 & 0 & 0 & 0 & 50 & 0 & 639 & 293 & 336 & 10 \\
\hline Co & $13(4)$ & 2673 & 26 & 7 & 92 & 102 & 89 & 1739 & 11 & 61 & 0 & 33 & 0 & 222 & 16 & 17 & 0 & 260 & 0 & 0 & 0 & 0 & 130 & 12 & 8 & 110 \\
\hline $\mathrm{Cp}$ & $10(3)$ & 8172 & 1041 & 867 & 147 & 1025 & 537 & 725 & 232 & 1138 & 9 & 689 & 20 & 313 & 387 & 145 & 272 & 272 & 311 & 0 & 42 & 0 & 3389 & 763 & 806 & 1821 \\
\hline $\mathrm{Cr}$ & $11(3)$ & 1323 & 36 & 14 & 39 & 214 & 114 & 343 & 12 & 170 & 0 & 41 & 4 & 51 & 26 & 12 & 20 & 136 & 62 & 0 & 31 & 0 & 568 & 61 & 15 & 491 \\
\hline $\mathrm{Cs}^{*}$ & $5(5)$ & 24529 & 9852 & 281 & 2094 & 889 & 3793 & 1441 & 138 & 1107 & 697 & 0 & 1519 & 804 & 348 & 273 & 568 & 369 & 282 & 0 & 18 & 56 & 70313 & 27972 & 27243 & 15099 \\
\hline $\mathrm{Cv}$ & $6(3)$ & 1832 & 21 & 34 & 124 & 75 & 54 & 183 & 14 & 117 & 29 & 31 & 62 & 761 & 0 & 25 & 56 & 98 & 23 & 0 & 126 & 0 & 898 & 325 & 369 & 204 \\
\hline $\mathrm{Dp}$ & $10(3)$ & 1717 & 49 & 29 & 22 & 147 & 435 & 145 & 25 & 91 & 97 & 53 & 0 & 39 & 430 & 8 & 15 & 104 & 26 & 0 & 0 & 0 & 887 & 13 & 76 & 798 \\
\hline $\mathrm{Ea}^{*}$ & $5(5)$ & 5094 & 49 & 37 & 3 & 108 & 47 & 225 & 4 & 630 & 70 & 42 & 0 & 0 & 3458 & 38 & 65 & 196 & 124 & 0 & 0 & 0 & 4493 & 652 & 157 & 3684 \\
\hline $\mathrm{Ec}$ & $8(5)$ & 642 & 19 & 16 & 3 & 41 & 82 & 85 & 42 & 87 & 44 & 17 & 3 & 57 & 0 & 6 & 7 & 4 & 4 & 0 & 125 & 0 & 1654 & 443 & 1201 & 10 \\
\hline $\mathrm{Ee}$ & $14(3)$ & 1375 & 25 & 60 & 287 & 113 & 54 & 182 & 62 & 230 & 41 & 74 & 0 & 25 & 116 & 17 & 11 & 46 & 11 & 0 & 21 & 0 & 163 & 47 & 47 & 69 \\
\hline En & $6(5)$ & 334 & 14 & 7 & 23 & 26 & 34 & 55 & 33 & 19 & 4 & 11 & 4 & 72 & 10 & 6 & 8 & 2 & 9 & 0 & 0 & 0 & 9 & 5 & 5 & 0 \\
\hline Es* & $4(4)$ & 1430 & 40 & 26 & 109 & 60 & 186 & 79 & 59 & 148 & 44 & 96 & 0 & 49 & 443 & 12 & 8 & 57 & 12 & 0 & 0 & 0 & 755 & 285 & 281 & 190 \\
\hline Eu & $14(2)$ & 294 & 5 & 6 & 11 & 28 & 23 & 41 & 4 & 13 & 15 & 13 & 21 & 36 & 45 & 3 & 5 & 16 & 2 & 0 & 6 & 0 & 216 & 84 & 111 & 21 \\
\hline Ev & $5(3)$ & 715 & 41 & 47 & 58 & 57 & 142 & 81 & 40 & 54 & 10 & 24 & 0 & 42 & 57 & 11 & 14 & 0 & 38 & 0 & 0 & 0 & 956 & 469 & 483 & 4 \\
\hline Ey & $12(1)$ & 273 & 6 & 2 & 7 & 15 & 15 & 35 & 9 & 57 & 0 & 12 & 0 & 56 & 1 & 2 & 9 & 16 & 8 & 0 & 23 & 0 & 267 & 113 & 142 & 12 \\
\hline $\mathrm{Fg}^{*}$ & 1 & 7346 & 129 & 219 & 520 & 528 & 1260 & 683 & 816 & 330 & 954 & 13 & 0 & 683 & 119 & 57 & 217 & 485 & 200 & 0 & 132 & 0 & 305 & 18 & 175 & 112 \\
\hline $\mathrm{Ga}$ & $6(1)$ & 355 & 6 & 2 & 6 & 26 & 14 & 67 & 4 & 19 & 3 & 0 & 25 & 118 & 14 & 3 & 12 & 15 & 14 & 0 & 5 & 0 & 103 & 51 & 35 & 17 \\
\hline $\mathrm{Ha}$ & $51(3)$ & 762 & 38 & 18 & 11 & 55 & 70 & 72 & 19 & 207 & 88 & 27 & 0 & 43 & 35 & 5 & 16 & 12 & 10 & 0 & 35 & 0 & 218 & 104 & 111 & 4 \\
\hline $\mathrm{Hc}$ & $13(2)$ & 1317 & 46 & 25 & 157 & 100 & 54 & 128 & 86 & 76 & 2 & 27 & 0 & 212 & 220 & 51 & 34 & 63 & 34 & 0 & 0 & 0 & 100 & 30 & 34 & 35 \\
\hline $\mathrm{He}$ & $8(3)$ & 867 & 45 & 82 & 47 & 66 & 77 & 72 & 104 & 97 & 47 & 32 & 7 & 45 & 30 & 19 & 28 & 45 & 13 & 0 & 11 & 0 & 286 & 134 & 137 & 15 \\
\hline $\mathrm{Hh}$ & $8(3)$ & 1946 & 75 & 149 & 62 & 121 & 112 & 137 & 572 & 272 & 12 & 46 & 17 & 71 & 72 & 29 & 92 & 0 & 105 & 0 & 0 & 0 & 487 & 146 & 153 & 188 \\
\hline $\mathrm{Hs}$ & $40(4)$ & 170 & 2 & 2 & 5 & 8 & 9 & 0 & 0 & 12 & 8 & 6 & 4 & 31 & 0 & 1 & 1 & 80 & 0 & 0 & 0 & 0 & 102 & 31 & 38 & 33 \\
\hline $\mathrm{La}^{*}$ & $4(4)$ & 3690 & 200 & 50 & 30 & 1000 & 180 & 890 & 20 & 290 & 0 & 0 & 20 & 60 & 70 & 70 & 190 & 200 & 330 & 0 & 0 & 90 & 202 & 68 & 14 & 120 \\
\hline Mn* & 11 (11) & 4472 & 184 & 160 & 893 & 278 & 375 & 280 & 130 & 220 & 217 & 35 & 455 & 203 & 150 & 75 & 123 & 381 & 165 & 0 & 149 & 0 & 252 & 116 & 134 & 2 \\
\hline $\mathrm{Mu}^{*}$ & 11 (11) & 3462 & 115 & 93 & 636 & 143 & 95 & 167 & 71 & 122 & 123 & 26 & 292 & 155 & 90 & 194 & 72 & 789 & 246 & 0 & 32 & 0 & 121 & 68 & 51 & 2 \\
\hline $\mathrm{Ng}^{*}$ & $3(3)$ & 576 & 25 & 15 & 9 & 30 & 34 & 39 & 4 & 26 & 0 & 8 & 0 & 49 & 299 & 6 & 15 & 0 & 17 & 0 & 0 & 0 & 317 & 3 & 27 & 287 \\
\hline Oc* & $3(3)$ & 1514 & 82 & 39 & 31 & 234 & 181 & 284 & 71 & 59 & 65 & 23 & 6 & 165 & 37 & 34 & 47 & 105 & 36 & 0 & 14 & 0 & 392 & 262 & 123 & 8 \\
\hline Pf* & $10(10)$ & 5770 & 140 & 80 & 60 & 270 & 510 & 230 & 30 & 890 & 0 & 0 & 40 & 150 & 3150 & 50 & 60 & 70 & 40 & 0 & 0 & 0 & 4084 & 267 & 1258 & 2559 \\
\hline Pg & $12(1)$ & 594 & 6 & 3 & 10 & 17 & 20 & 32 & 41 & 19 & 29 & 3 & 0 & 103 & 222 & 5 & 8 & 38 & 26 & 0 & 11 & 0 & 124 & 58 & 63 & 3 \\
\hline $\mathrm{Pm}^{*}$ & $9(9)$ & 2020 & 70 & 30 & 10 & 140 & 60 & 210 & 20 & 180 & 220 & 0 & 60 & 80 & 750 & 20 & 50 & 40 & 80 & 0 & 0 & 0 & 4900 & 980 & 267 & 3653 \\
\hline $\mathrm{Pp}$ & 14 (2) & 307 & 7 & 7 & 17 & 25 & 54 & 45 & 4 & 35 & 12 & 17 & 5 & 40 & 5 & 4 & 10 & 2 & 1 & 0 & 16 & 0 & 434 & 151 & 155 & 128 \\
\hline Ps & $18(2)$ & 138 & 2 & 1 & 2 & 7 & 6 & 17 & 2 & 13 & 3 & 6 & 5 & 18 & 14 & 0 & 1 & 35 & 6 & 0 & 0 & 0 & 7 & 4 & 2 & \\
\hline
\end{tabular}




\begin{tabular}{|c|c|c|c|c|c|c|c|c|c|c|c|c|c|c|c|c|c|c|c|c|c|c|c|c|c|c|}
\hline \multirow{3}{*}{$\begin{array}{l}\text { Plant } \\
\text { species }^{1}\end{array}$} & \multirow{3}{*}{$\begin{array}{c}\mathrm{N} \\
\left(\mathrm{N}^{\prime}\right)^{2}\end{array}$} & \multicolumn{21}{|c|}{ Amino acids } & \multicolumn{4}{|c|}{ Sugars ${ }^{6}$} \\
\hline & & $\begin{array}{l}\text { Total } \\
\text { AAs }^{3}\end{array}$ & $\mathrm{Asp}^{4}$ & Glu & Asn & Ser & $\begin{array}{l}\text { His }+ \\
\mathrm{Gln}^{5}\end{array}$ & $\begin{array}{l}\text { Gly }+ \\
\text { Thr }^{5}\end{array}$ & $\operatorname{Arg}$ & $\begin{array}{l}\mathrm{Tyr}+ \\
\mathrm{Ala}^{5}\end{array}$ & Gaba & Trp & Met & Val & Phe & Ile & Leu & Orn & Lys & H-Ser & Unknown & $\beta$-Ala & $\begin{array}{c}\text { Total } \\
\text { sugars }\end{array}$ & Glucose & Fructose & Sucrose \\
\hline & & \multicolumn{21}{|c|}{ pmoles/flower } & \multicolumn{4}{|c|}{ nmoles/flower } \\
\hline Pso & $15(3)$ & 480 & 26 & 13 & 34 & 47 & 17 & 68 & 5 & 69 & 25 & 13 & 7 & 37 & 22 & 5 & 13 & 34 & 10 & 0 & 36 & 0 & 707 & 40 & 49 & 618 \\
\hline $\mathrm{Pv}$ & $8(2)$ & 1296 & 53 & 36 & 79 & 60 & 94 & 90 & 131 & 124 & 0 & 33 & 0 & 52 & 392 & 19 & 19 & 26 & 18 & 0 & 69 & 0 & 815 & 376 & 435 & 4 \\
\hline $\mathrm{Ra}$ & 5 (3) & 1489 & 24 & 45 & 78 & 100 & 275 & 103 & 62 & 113 & 108 & 125 & 0 & 25 & 82 & 11 & 18 & 104 & 79 & 0 & 139 & 0 & 794 & 76 & 75 & 643 \\
\hline $\operatorname{Re}$ & $5(3)$ & 3024 & 53 & 51 & 21 & 243 & 326 & 270 & 221 & 287 & 0 & 54 & 0 & 65 & 1167 & 23 & 56 & 113 & 50 & 0 & 23 & 0 & 1283 & 457 & 436 & 390 \\
\hline $\mathrm{Rg}$ & $8(3)$ & 2318 & 19 & 25 & 34 & 81 & 71 & 203 & 171 & 812 & 0 & 18 & 0 & 178 & 19 & 4 & 37 & 124 & 9 & 0 & 515 & 0 & 3418 & 1146 & 2047 & 225 \\
\hline Ro & 4 (4) & 2599 & 80 & 152 & 38 & 243 & 365 & 263 & 111 & 285 & 0 & 21 & 187 & 118 & 90 & 24 & 198 & 344 & 0 & 0 & 80 & 0 & 377 & 155 & 180 & 41 \\
\hline $\mathrm{Rp}$ & 20 (3) & 433 & 25 & 27 & 3 & 44 & 62 & 50 & 16 & 70 & 0 & 22 & 0 & 61 & 3 & 6 & 15 & 3 & 4 & 0 & 21 & 0 & 202 & 78 & 78 & 46 \\
\hline $\mathrm{Sa}$ & 8 (3) & 567 & 20 & 9 & 7 & 38 & 76 & 60 & 4 & 46 & 26 & 16 & 0 & 143 & 18 & 10 & 4 & 39 & 45 & 0 & 9 & 0 & 361 & 155 & 157 & 48 \\
\hline $\mathrm{Sb}^{*}$ & 7 (7) & 3200 & 0 & 0 & 0 & 50 & 40 & 120 & 10 & 40 & 60 & 0 & 20 & 60 & 2670 & 20 & 20 & 30 & 60 & 0 & 0 & 0 & 752 & 244 & 109 & 399 \\
\hline $\mathrm{Sc}$ & 9 (4) & 324 & 12 & 4 & 23 & 37 & 8 & 63 & 8 & 43 & 0 & 7 & 0 & 53 & 10 & 4 & 13 & 15 & 5 & 0 & 17 & 0 & 321 & 148 & 121 & 52 \\
\hline $\mathrm{Sg}$ & 8 (3) & 6874 & 127 & 94 & 1715 & 462 & 1375 & 473 & 79 & 744 & 0 & 76 & 7 & 224 & 202 & 83 & 116 & 502 & 419 & 0 & 175 & 0 & 922 & 313 & 319 & 290 \\
\hline $\mathrm{Si}^{*}$ & 4 (4) & 395 & 4 & 3 & 0 & 16 & 5 & 42 & 13 & 139 & 0 & 6 & 0 & 36 & 2 & 9 & 5 & 55 & 31 & 0 & 29 & 0 & 178 & 91 & 85 & 3 \\
\hline $\mathrm{Sj}^{*}$ & $6(6)$ & 4280 & 90 & 30 & 20 & 360 & 120 & 370 & 30 & 690 & 0 & 0 & 20 & 110 & 2060 & 20 & 60 & 180 & 120 & 0 & 0 & 0 & 391 & 62 & 97 & 232 \\
\hline So & $5(3)$ & 1866 & 54 & 130 & 56 & 136 & 183 & 222 & 57 & 238 & 328 & 55 & 44 & 91 & 34 & 17 & 21 & 41 & 25 & 0 & 134 & 0 & 603 & 283 & 315 & 5 \\
\hline $\mathrm{St}^{*}$ & $5(5)$ & 4900 & 170 & 100 & 100 & 360 & 330 & 390 & 330 & 310 & 40 & 0 & 140 & 350 & 1830 & 180 & 80 & 80 & 90 & 0 & 20 & 0 & 6614 & 974 & 1406 & 4233 \\
\hline $\mathrm{Su}$ & $8(3)$ & 633 & 15 & 18 & 114 & 65 & 44 & 39 & 25 & 74 & 10 & 124 & 0 & 19 & 17 & 10 & 25 & 0 & 34 & 0 & 0 & 0 & 165 & 83 & 82 & 0 \\
\hline Sy* & $10(10)$ & 17240 & 150 & 60 & 130 & 340 & 230 & 380 & 400 & 640 & 0 & 0 & 590 & 0 & 13780 & 70 & 70 & 140 & 220 & 0 & 40 & 0 & 2048 & 155 & 384 & 1509 \\
\hline $\mathrm{Ta}$ & 15 (4) & 166 & 7 & 3 & 0 & 17 & 8 & 32 & 5 & 21 & 3 & 7 & 0 & 45 & 7 & 1 & 10 & 0 & 0 & 0 & 0 & 0 & 125 & 50 & 58 & 17 \\
\hline Tc* & $8(8)$ & 2570 & 60 & 10 & 0 & 260 & 60 & 230 & 10 & 210 & 0 & 30 & 70 & 140 & 770 & 20 & 30 & 200 & 460 & 0 & 10 & 0 & 348 & 51 & 176 & 121 \\
\hline $\mathrm{Td}^{*}$ & $3(3)$ & 9310 & 560 & 130 & 20 & 110 & 80 & 130 & 0 & 1270 & 0 & 0 & 80 & 40 & 6660 & 50 & 60 & 40 & 40 & 0 & 30 & 10 & 3262 & 559 & 692 & 2011 \\
\hline $\mathrm{Te}^{*}$ & $10(10)$ & 2650 & 350 & 30 & 20 & 150 & 100 & 160 & 10 & 390 & 40 & 30 & 20 & 90 & 1030 & 10 & 40 & 70 & 80 & 0 & 30 & 0 & 1241 & 116 & 350 & 775 \\
\hline $\mathrm{Tg}$ & $5(3)$ & 869 & 13 & 17 & 5 & 50 & 32 & 66 & 7 & 82 & 24 & 26 & 0 & 51 & 400 & 6 & 30 & 0 & 60 & 0 & 0 & 0 & 1223 & 513 & 690 & 20 \\
\hline Th & $10(2)$ & 346 & 10 & 9 & 9 & 49 & 18 & 76 & 11 & 32 & 23 & 35 & 0 & 41 & 2 & 3 & 7 & 2 & 19 & 0 & 0 & 0 & 7 & 2 & 2 & 4 \\
\hline $\mathrm{Tm}$ & 24 (3) & 216 & 9 & 8 & 11 & 19 & 13 & 26 & 3 & 38 & 0 & 16 & 0 & 29 & 4 & 2 & 4 & 13 & 11 & 0 & 10 & 0 & 382 & 147 & 166 & 69 \\
\hline $\mathrm{Tp}$ & 14 (3) & 536 & 23 & 13 & 18 & 60 & 63 & 81 & 13 & 77 & 10 & 19 & 0 & 74 & 5 & 6 & 17 & 30 & 14 & 0 & 15 & 0 & 393 & 168 & 185 & 40 \\
\hline Ts* & $3(3)$ & 364 & 17 & 14 & 53 & 40 & 19 & 53 & 7 & 50 & 0 & 6 & 0 & 50 & 5 & 4 & 7 & 18 & 17 & 0 & 6 & 0 & 246 & 79 & 89 & 78 \\
\hline Um* & 4 (4) & 2749 & 18 & 20 & 27 & 68 & 180 & 148 & 13 & 85 & 61 & 29 & 0 & 58 & 1994 & 33 & 16 & 0 & 0 & 0 & 0 & 0 & 2416 & 1376 & 1041 & 0 \\
\hline \multicolumn{27}{|l|}{ Mean } \\
\hline plant & & 2908 & 236 & 71 & 157 & 184 & 265 & 309 & 78 & 277 & 73 & 45 & 56 & 126 & 699 & 36 & 54 & 102 & 67 & 2 & 71 & 3 & 2478 & 869 & 906 & 703 \\
\hline
\end{tabular}

${ }_{2}^{1}$ Except few cases of relatively high nectar yielding plants (indicated with $*$ ), in ca $2 / 3$ of the plant species several flowers were pooled for analysis

${ }^{2}$ The amounts (pmoles flower ${ }^{-1}$ ) given in the table are means over N' runs (in parentheses), calculated on the basis of the mean values per run. $\mathrm{N}$ is the total number of flowers analyzed ${ }^{3}$ Total value is the sum of all particular AAs (pmoles flower ${ }^{-1}$ ) or sugars (nmole flower ${ }^{-1}$ ) within a plant species. Mean value/plant is the average of all values (AAs, sugars; particular or total) of all plant species within the community

4 The order of AA presentation follows the compound appearance in the chromatograms. Abbreviations are: Ala-alanine, Arg-arginine, Asn-asparagine, Asp-aspartic acid, $\beta$-Ala- $\beta$ alanine, Gaba- $\gamma$-aminobutyric acid, Gln-glutamine, Glu-glutamic acid, Gly-glycine, His-histidine, H-ser-homoserine, Ile-isoleucine, Leu-leucine, Lys-lysine, Met-methionine, Ornornithine, Phe-phenylalanine, Ser-serine, Trp-tryptophane, Tyr-tyrosine, Val-valine. Plant abbreviations are as in Table 1

Because in most chromatograms they were inseparable, the groups: His + Gln and "unknown" were calculated as His equivalent; Gly + Thr as Thr equivalent; Tyr + Al as Tyr equivalent; and $\beta$-Ala as Ala equivalent. Under "unknown" the total amount of three different unknown compounds are grouped

Data from Petanidou (2005) 
the p-p food web of the community (Petanidou 1991, Petanidou and Ellis 1996). Each of the remaining particular AAs contributed with less than $20 \%$ to the total AA composition in all the plant families studied.

\section{Phylogenetic and ecological constraints}

Using Wilk's lambda test criterion in a multivariate ANOVA, in which the AA\&S percentages in the nectar of each plant species were taken as dependent variables, and plant taxonomic group, life form and season as factors (fixed, discrete), we found that only plant taxonomic group has a significant, although quite weak, effect, whereas life form and season no discernable influence (Table 3). Scheffe's post hoc tests revealed that weak difference were discernable in asparagine between lamiids and fabids and glutamic acid between malvids and campanulids. Remarkably, phenylalanine, the most abundant AA, and all the "three abundant" sugars did not vary notably among any of the categories.

\section{Pollinators' preferences}

Figure 1 and 2 give the results of PCAs for flowervisiting guilds and bee families respectively. Note that because of the generally high phenylalanine percentages most of the other AAs are pushed to one side of the plots. The majority of the bee families and some guilds, most clearly the long tongued bees, and among these the Megachilidae and Anthophoridae, are centred in the phenylalanine-rich part of the plot. Gamma-aminobutyric acid (GABA) seems to have a similar, however a weaker and lees focused effect spread among a wide range of insect guilds. In general it is surprising that the behaviour of the pollinator guilds is so uniform, with wasps, short tongued bees, other Diptera and Coleoptera falling in one overlapping cluster. It is also striking that the long tongued bees, the most specialised pollinators of all, stand the most isolated in their preference for a an extremely high-phenylalanine nectar in which sucrose is the dominant sugar and nectar volume has some effect. Other families and guilds, most clearly the Syrphidae, prefer a "broth" high in phenylalanine, but also containing many other AAs, like tryptophane, arginine, glutamic acid and histidine-glutamine in hexose-rich nectars. AAs that seem to be avoided by some insect groups are asparagine (by all guilds and bee families) and glycine-threonine, $\mathrm{H}$-serine, serine, $\beta$-alanine, valine, leucine (by most bee families).

To analyse this in somewhat more detail we performed stepwise multiple regressions of the number of visitors per family and guild upon the nectar values (i.e. AA\&S percentages and nectar volume; Table 4). From the table, like from the PCAs, it is apparent that asparagine and $\mathrm{H}$ serine have a generally negative partial coefficient, while few others (methionine, valine, leucine) have negative effects on individual insect groups. Tryptophane has both a negative (viz. for long tongued bees and Anthophoridae), and a positive effect (viz. for Coleoptera and Syrphidae). Phenylalanine and GABA were the only AAs having a generally positive effect on insect groups, especially for long tongued bees with partial effect on Megachilidae (phenylalanine) and Anthophoridae (GABA).

As the PCA plots show, phenylalanine is loosely associated with sucrose-rich nectars, while most AAs of the "broth" predominantly occur in low volume, hexoserich nectars. Voluminous nectars apparently are favoured by Megachilidae, while most other guilds and families seem to prefer low-volume, i.e. concentrated, nectars. We also calculated multiple regressions of the number of visitors per guild and family upon the three main parameters of nectar: volume, total AA content and total sugar content (Table 5). The table shows that among all insect guilds only long tongued bees, and in particular Anthophoridae, showed a significant dependence upon the total AA content of nectars. Total sugar had no effect at all, whereas nectar volume had only negative impacts, especially on flies and wasps. Lepidoptera were not significantly associated with any of the three parameters.

\section{Discussion}

\section{Phenylalanine: an important amino acid in the phryganic nectars}

Although in low concentration in floral nectars, particularly when compared to sugars, AAs are commonly present in the floral nectars of phrygana, where they were found to be represented by 22 AAs or AA groups (Petanidou et al. 1996, Petanidou 2005). Among them phenylalanine dominated in many respects. The dominance was particularly obvious within most members of the Lamiaceae family, where it contributed by over $50 \%$ to the total AA amount. Such extreme quantities of phenylalanine were also found in the nectars of Satureja thymbra and Salvia fruticosa in Israel (phenylalanine accounting for $71 \%$ and $52 \%$ of the total AAs, respectively). However, phenylalanine content in the nectars of another two species of the same study, viz. Thymus capitatus and Rosmarinus officinalis, was at community average levels (Dafni et al. 1988).

Moreover phenylalanine, together with GABA, was the only AA in the phryganic nectars that is clearly attractive to certain insect guilds (i.e. long tongued bees; Fig. 1) and families (i.e. Megachilidae, Anthophoridae; Fig. 2). Other families and guilds, most clearly the Syrphidae, prefer a hexose-rich "broth" which is high in phenylalanine and rich in other AAs, in particularly 
Table 3. Results of a MANOVA with nectar AA and sugar percentages, as well as volume as dependent variables and plant taxonomic group, life form and flowering season as independent variables, using Wilk's lambda test criterion. All data were transformed $\left(\mathrm{x}^{\prime}=\ln (\mathrm{x}+1)\right)$. In post hoc results the groups with the highest values are given first.

\begin{tabular}{lcccccc}
\hline & Lambda & P & F & df, error df & $\begin{array}{c}\text { Dependent (nectar) and } \\
\text { independent variable groups } \\
\text { responsible for the difference }{ }^{1}\end{array}$ & $\begin{array}{c}\text { Pairs of differring independent } \\
\text { variable groups (Scheffe's } \\
\text { post hoc results) }\end{array}$ \\
\hline $\begin{array}{l}\text { Constant } \\
\text { Plant taxonomy group }\end{array}$ & 0.004 & 0.000 & 374 & 24,32 & & \\
& 0.034 & 0.042 & 1.34 & 120,162 & Asn-fabids $(\mathrm{P}<0.0176)$ & fabids-lamiids $(\mathrm{P}<0.0206)$ \\
Life form & 0.152 & 0.227 & 1.18 & 72,96 & Glu-malvids $(\mathrm{P}=0.0013)$ & malvids-campanulids $(\mathrm{P}<0.0358)$ \\
Season & 0.125 & 0.086 & 1.35 & 72,96 & & \\
\hline
\end{tabular}

${ }^{1}$ We give only those pairs that finally give significant Scheffés post hoc results

tryptophane. Interestingly, short tongued bees lie within these guilds, being comparable in behaviour with wasps rather than longed tongued bees (cf. Table 4). Apidae differentiate from the other long tongued bees, i.e. Megachilidae and Anthophoridae, probably because they visit systematically and almost indiscriminately the majority of the available flora within the community (Petanidou 1991).

Whereas we have no reference from other studies on the effect of GABA and asparagine, the above results are in agreement with previous studies showing that phenylalanine is an AA of particular importance in the context of pollination ecology. Although highly variable in floral nectars, phenylalanine is one of the most common AAs in floral honeys (Bose and Battaglini 1978), one of the ten essential AAs for honeybees (Groot 1953, Chapman 1983, Dafni and Kevan 1994) and a precursor of the specific aroma component phenyl-ethanol (Thawley

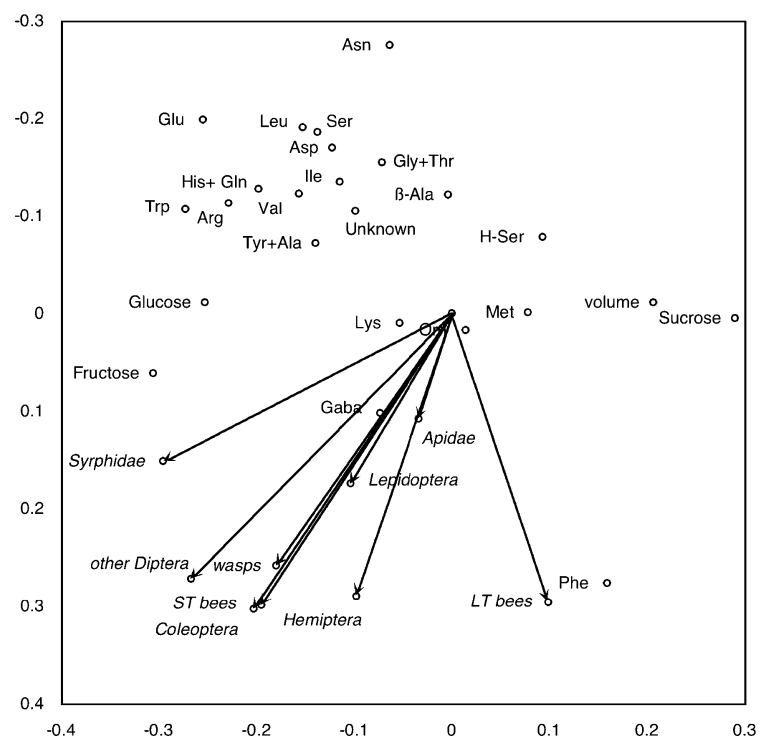

Fig. 1. Principal components analysis (PCA) run on the basis of the nectar attributes of the 67 plant species (AAs and sugar percentages; nectar volume) and all insect species per guild visiting each plant species. First and second component axes account for $15.4 \%$ and $13.2 \%$ of the total variance. Origin is at $(0,0)$.
1969). Most important, phenylalanine has been found to be the only AA with a strong phagostimulatory effect on honeybees at the highest concentrations tested (Inouye and Waller 1984). The latter finding is very relevant to our results, implying that bees may detect and respond to differences in concentrations of some AAs in solution (Inouye and Waller 1984), as do hummingbirds (Hainsworth and Wolf 1976).

In contrast with the phrygana, phenylalanine had a significantly lower concentration (M-W U test, $\mathrm{P}<$ 0.0001 ) in 30 British plant species analysed by Gardener and Gillman (2001). We attribute the dominance of phenylalanine in the Mediterranean to the high number of bees, especially longed tongued ones, and we argue that in the Mediterranean such bees might have acted as crucial selective factors for phenylalanine-rich nectars (Michener 1979, 2000, O’Toole and Raw 1991, Petanidou and Ellis 1993, 1996, Dafni and O'Toole 1994,

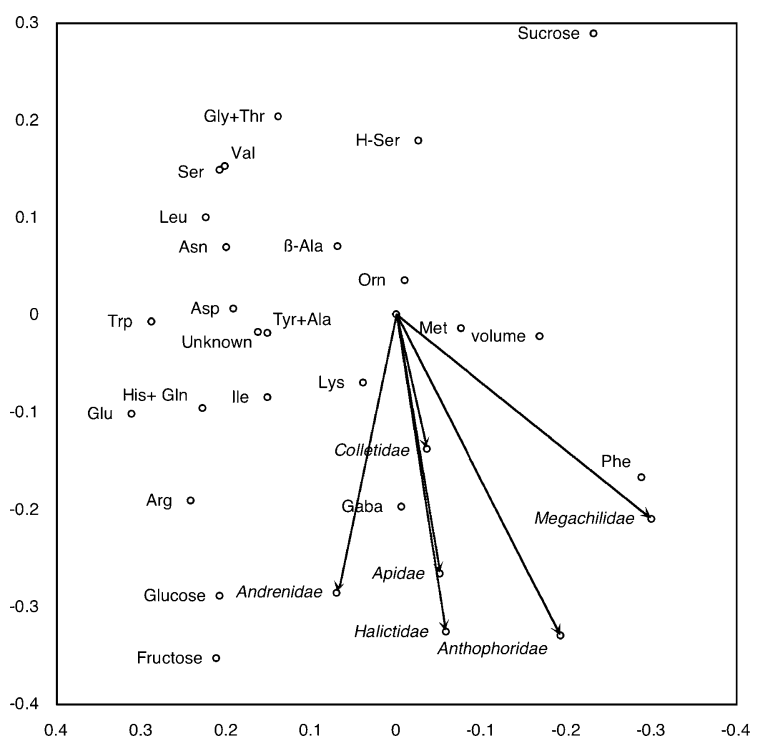

Fig. 2. Principal components analysis (PCA) run on the basis of the nectar attributes of the 67 plant species (AAs and sugar percentages; nectar volume) and all visiting bee species shown per family. First and second component axes account for $16.5 \%$ and $10.2 \%$ of the total variance. Origin is at $(0,0)$. 
Table 4. Relationship of different flower visiting insect groups (guilds and bee families) to different nectar parameters of the floral nectars of the phryganic plants (AAs, major sugars, nectar volume) visited by each group. We give multiple regression results (adjusted overall $\mathrm{R}^{2}$ and partial coefficients) of flower visitors (numbers of visitor species of a certain insect group per plant species) over the proportions of different AAs ( $\%$ of each AA over the total amount of AAs) and the proportions of different sugars ( $\%$ over the total amount of sugars) contained in the nectars of the same plant species. The analysis was made on 67 plant species. All data were transformed $\left(\mathrm{x}^{\prime}=\ln (\mathrm{x}+1)\right)$. We give only significant values: *: $\mathrm{P}<0.05, * *$ : $0.05<\mathrm{P}<0.01, * * *: 0.01<\mathrm{P}<0.001$

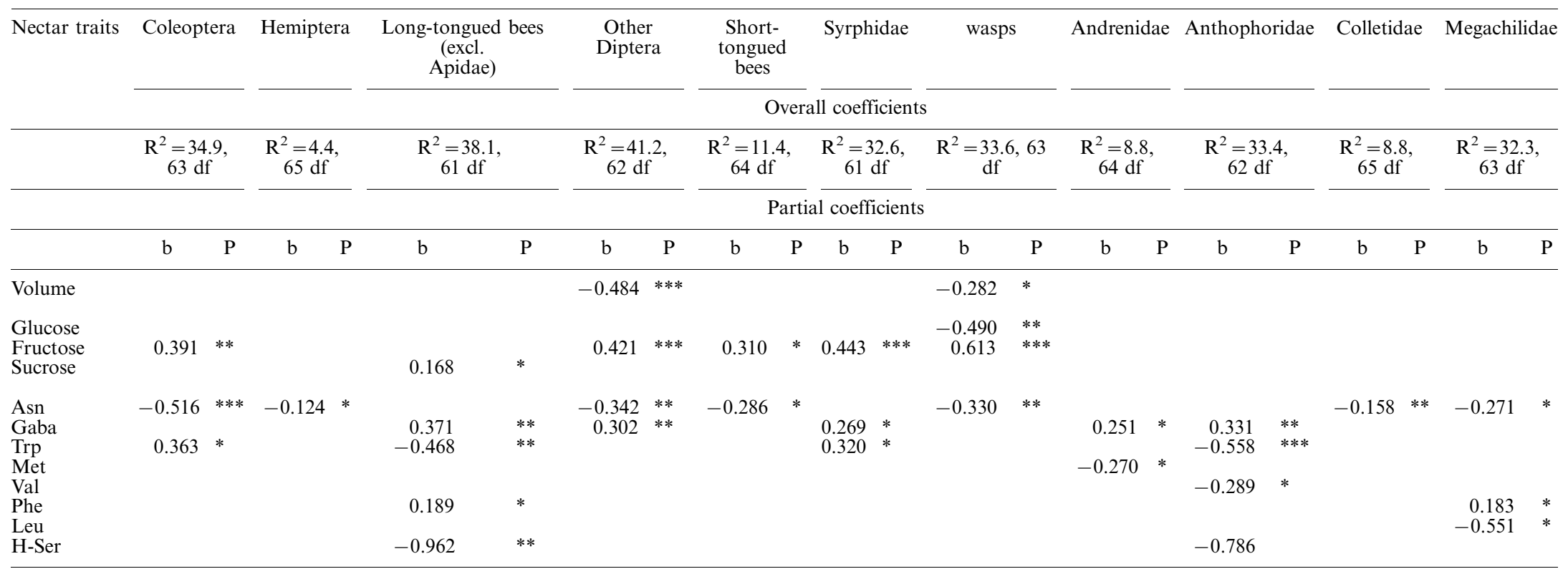




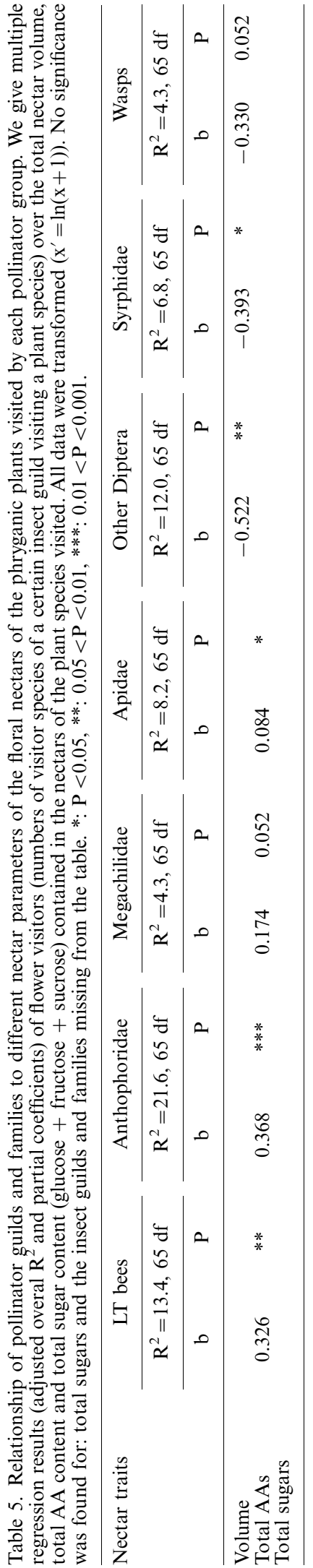

Petanidou and Potts 2006). Similarly, the dominance of phenylalanine in the Lamiaceae may be explained by the highly melittophilous character of this family, hence selected by the high number of bees visiting it (Petanidou and Vokou 1993). Such coevolution between long tongued bees and phenylalanine-rich nectars was less prominent in Thymus capitatus and the cultivated Rosmarinus officinalis studied in Israel by Dafni et al. (1988). Interestingly, T. capitatus had a low phenylalanine score also within the Lamiaceae of the phrygana studied (second lowest, second only to Lamium amplexicaule; Table 2). This is not surprising, because thyme flowers in a competition-free period (summer) in both communities, in which it functions as a "pollinator sink" visited by a large array of insects (viz. 123 species in the study phrygana, consisting of bees (24\%), wasps $(18 \%)$, flies $(20 \%)$ and other insect groups; Petanidou 2004, Petanidou and Potts 2006). With a very extended flowering period in wintertime and visited mainly by honeybees (T. Petanidou, pers. obs.) R. officinalis seems also to face no competition for pollination - a major hint for coevolution at the community level. The case of $L$. amplexicaule might be partly explained by the cleistogamic character of its flowers (Lord 1982, Petanidou 1991).

On basis of the above we argue that phenylalanine constitutes a very important compound of the phryganic nectars and pollinator diet. Its importance appears to be highest for bees, especially the long tongued ones, and among them Megachilidae. What is the specific role phenylalanine plays in the floral nectars of phrygana? One possibility is that, by being one of the essential AAs for bees (Groot 1953), phenylalanine constitutes an AA source for bees, in addition to the pollen collected by the females. However, because no other essential AA in the floral nectars of phrygana has a comparable effect on bees as phenylalanine (GABA is a non-essential AA), we believe that a better explanation is offered by the strong phagostimulatory effect of phenylalanine that was found or discussed in earlier studies (Inouye and Waller 1984, Gardener and Gillman 2002). By acting as a phagostimulant phenylalanine constitutes a major coevolutionary mechanism in shaping the nectars within the Mediterranean area, with long tongued bees, and especially Megachilids acting as the main selective agents. In this respect, the evident significance of GABA, a neurotransmitter that is "absolutely dependent on sodium and chloride" (Keynan and Kanner 1988, Wolfersberger 2000), in the nectars of phrygana seems to be highly relevant. On the basis of the above, we argue that it may not be GABA alone, as evidenced by Inouye and Waller (1984), but its combination with $\mathrm{NaCl}-$ or even $\mathrm{NaCl}$ only that constitutes an important nectar trait that bees and other anthophilous insects are allured by. The combined phagostimulatory effect of $\mathrm{GABA}$ and $\mathrm{NaCl}$, associated 
with flowers yielding relatively low nectar (Fig. 1, 2) is certainly an interesting issue that merits special attention in future studies.

\section{What does shape floral nectar composition?}

We have evidence to support the hypothesis that nectar composition (AA\&S + nectar volume) in phrygana appears to have been weakly shaped by phylogenetic relationships, not by ecological constraints. However, the most decisive players in shaping chemical composition in the floral nectars of these habitats appear to have been the flower visiting insects, primarily the long tongued bees and especially Megachilids in the supreme role of selective agents. This emphasises the importance of coevolution in the process of evolution at the community level in the Mediterranean area, which appears more overwhelming than the overriding influence of Mediterranean climate. In fact, climate has been found to be extremely important in shaping many other pollination and nectar characteristics detected independently (e.g. flowering, nectary size, nectar secretion; Petanidou et al. 1995, 2000).

Recent findings on the biology of other insects uphold the conclusion that coevolution between flower visiting insects and AA\&S composition of nectar is widespread, although this concerns mainly sugars, rather than AAs (reviewed by Petanidou 2005). As to AAs, and according to the "consensus view", such a coevolution should especially apply to pollinators that have no alternative nitrogen resources, such as butterflies and moths (Baker and Baker 1986, Gardener and Gillman 2002). Indeed, experimental work on butterflies has shown that Pieris rapae females prefer nectar containing AAs over sugar-only nectars (Alm et al. 1990), Pieris brassicae females select for nectar rich in sucrose and AAs (Romeis and Wackers 2000), while females of the species Inachis io, Pieris napi and Araschnia levana detect and prefer nectar with high AA content, especially when they have been deprived of AAs during their larval stage (Erhardt and Rusterholz 1998, Mevi-Schutz and Erhardt 2003, 2004). The concluding view that floral nectar AAs favour butterfly fecundity and fitness (Mevi-Schutz and Erhardt 2005) has been criticized repeatedly by other authors suggesting that most of the egg AAs needed by female butterflies originate from nectar sugars, whereas essential AAs entirely from the larval diet (O'Brien et al. 2002, Jervis and Boggs 2005). Evidently our results do not support the above "consensus view", as no significant relation of Lepidoptera with any of the nectar variables tested was revealed (Fig. 1, Table 4, 5). An explanation can be that as a pollinator guild, Lepidoptera are relatively unimportant in the Mediter- ranean, therefore non influential in shaping nectar traits, at least much less important than bees (Herrera 1987).

A major result of this study is also that solitary bees, especially long tongued bees, respond positively to the total AAs of nectar in phrygana (Table 5). As far as we know, since the historical paper by Baker and Baker (1986), solitary bees alone have never been considered as selective agents for high AA content of nectar. According to Gardener and Gillman (2002) honeybees and flies show a preferential response to AAs contained in nectarmimicking solutions, whereas nectar-feeding ants also show some preference for AAs (Bluthgen and Fiedler 2004). Tropical bees are exempted from this rule. For instance, the tropical bee Trigona hockingsi shows no preference for AAs in artificial nectars (Gardener et al. 2003). Similarly, AA solutions did not affect attractiveness to tropical bees of the subfamilies Euglossini, Meliponini, and Centridini as much as sugar solutions (Roubik et al. 1995). These results can be explained by the differential nectar choice of tropical bees in general, as tropical systems are dominated by species secreting relatively dilute nectar, therefore sugars may play a greater role in nectar choice rather than AAs (Cruden et al. 1983, Petanidou and Smets 1995). Yet, by having high quantities of nectar at their disposal, by the end of the day tropical bees get the AA quantity required to cover their needs.

Finally, why AAs appear more important than sugars in shaping the $p-p$ interaction web within a community characterised by an outstanding melittophily? As inferred by Gardener and Gillman (2002) sugars "by far dominate the taste of nectars" because they represent the most abundant compound therein. But by being so dominant, sugars are expected not to constitute the main discriminatory compound for the response of the nectar consumers in phrygana, which is the case in other habitats (Gardener and Gillman 2002). This can be explained as in dry and hot Mediterranean habitats nectars have very high sugar concentrations (Petanidou and Smets 1995), therefore with a sweet taste that is generally too "strong". In such habitats, dominated by thick nectars, it is probable that AAs constitute the discriminant taste and take over as major taste stimulators and contributors to the overall nectar palatability. In this respect, the high contribution of phenylalanine, a well known phagostimulant (Inouye and Waller 1984) and the significant presence of GABA, a NaCl-dependent AA (Wolfersberger 2000), in the nectars of phrygana, appear to be highly meaningful.

Acknowledgements - The study was supported by an EC grant made available to Theodora Petanidou (DG VI-contract AIR3BM92-004). We acknowledge "I. and A. Diomedes Botanical Garden of Athens University" for supporting the extensive 
fieldwork, E. Nackaerts for technical assistance on amino acid analyses and Jane Memmott and Peter Davies for their invaluable comments on earlier versions of this manuscript.

\section{References}

A. P. G. (Angiosperm Phylogeny Group). 1998. An ordinal classification for the families of flowering plants. - Ann. Miss. Bot. Gard. 85: 531-553.

Alm, J., Ohnmeiss, T. E., Lanza, J. et al. 1990. Preference of cabbage white butterflies and honey bees for nectar that contains amino acids. - Oecologia 84: 53-57.

Baker, H. G. and Baker, I. 1973a. Amino acids in nectar and their evolutionary significance. - Nature 241: 543-545.

Baker, H. G. and Baker, I. 1973b. Some anthecological aspects of the evolution of nectar-producing flowers, particularly amino acid production in nectar. - In: Heywood, V. H. (ed), Taxonomy and ecology. Academic Press, pp. 243-264.

Baker, H. G. and Baker, I. 1975. Studies of nectar constitution and pollinator-plant coevolution. - In: Gilbert, L. E. and Raven, P. H. (eds), Coevolution of animals and plants. Univ. of Texas Press, pp. 100-140.

Baker, H. G. and Baker, I. 1979. Sugar ratios in nectars. - Phytochem. Bull. 12: 43-45.

Baker, H. G. and Baker, I. 1982. Chemical constituents of nectar in relation to pollination mechanisms and phylogeny. - In: Nitecki, M. H. (ed.), Biochemical aspects of evolutionary biology. Proc. 4th Ann. spring Syst. Symp. Univ. of Chicago Press, pp. 131-172.

Baker, H. G. and Baker, I. 1983. Floral nectar sugar constituents in relation to pollinator type. - In: Jones, C. E. and Little, R. J. (eds), Handbook of experimental pollination ecology. Van Nostrand-Reinhold, pp. 117-141.

Baker, H. G. and Baker, I. 1986. The occurrence and significance of amino acids in floral nectar. - Plant Syst. Evol. 151: $175-186$.

Baker, H. G. and Baker, I. 1990. The predictive value of nectar chemistry to the recognition of pollinator types. - Isr. J. Bot. 39: $157-166$.

Baker, H. G., Opler, P. A. and Baker, I. 1978. A comparison of the amino acid complements of floral and extrafloral nectars. - Bot. Gaz. 139: 322-332.

Bluthgen, N. and Fiedler, K. 2004. Preferences for sugars and amino acids and their conditionality in a diverse nectarfeeding ant community. - J. Anim. Ecol. 73: 155-166.

Bose, G. and Battaglini, M. 1978. Gas chromatographic analysis of free and protein amino acids in some unifloral honeys. - J. Apic. Res. 17: 152-166.

Chapman, R. F. 1983. The insects: structure and function. - Harvard Univ. Press.

Corbet, S. A. 1990. Pollination and the weather. - Isr. J. Bot. 39: $13-30$.

Cruden, R. W., Hermann, S. M. and Peterson, S. 1983. Patterns of nectar production and plant-pollinator coevolution. - In: Bentley, B. and Elias, T. (eds), The biology of nectarines. Columbia Univ. Press, pp. 80-125.

Dafni, A. and Kevan, P. G. 1994. A hypothesis on complementary amino acids in nectar. - Evol. Theory 10: 259-260.

Dafni, A. and O'Toole, C. 1994. Pollination syndromes in the Mediterranean: generalizations and peculiarities. - In: Arianoutsou, M. and Groves, R. H. (eds), Plant-animal interactions in Mediterranean-type systems. Kluwer, pp. $125-135$.

Dafni, H., Lensky, Y. and Fahn, A. 1988. Flower and nectar characteristics of nine species of Lamiaceae and their influence on honeybee visits. - J. Apic. Res. 27: 103-114.

Erhardt, A. 1991. Nectar sugar and amino acid preference of Battus philenor (Lepidoptera, Papilionidae). - Ecol. Entomol. 16: $425-434$.
Erhardt, A. 1992. Preferences and non preferences for nectar constituents in Ornithoptera priamus poseidon (Lepidoptera, Papilionidae). - Oecologia 90: 581-585.

Erhardt, A. and Rusterholz, H. P. 1998. Do peacock butterflies (Inachis io L.) detect and prefer nectar amino acids and other nitrogenous compounds? - Oecologia 117: 536-542.

Gardener, M. C. and Gillman, M. R. 2001. Analyzing variability in nectar amino acids: composition is less variable than concentration. - J. Chem. Ecol. 27: 2545-2558.

Gardener, M. C. and Gillman, M. P. 2002. The taste of nectar a neglected area of pollination ecology. - Oikos 98: 552557.

Gardener, M. C., Rowe, R. J. and Gillman, M. P. 2003. Tropical bees (Trigona hockingsi) show no preference for nectar with amino acids. - Biotropica 35: 119-125.

Gottsberger, G., Arnold, T. and Linskens, H. F. 1989. Are amino acid and sugar concentration correlated in floral nectar? - Kon. Ned. Akad. Wet. Proc. C 92: 461-464.

Gottsberger, G., Arnold, T. and Linskens, H. F. 1990. Variations in floral nectar amino acids with aging of flowers, pollen contamination, and flower damage. - Isr. J. Bot. 39: 167176.

Gottsberger, G., Schrauwen, J. and Linskens, H. F. 1984. Amino acids and sugars in nectar, and their putative evolutionary significance. - Plant Syst. Evol. 145: 55-77.

Groot, A. P. de. 1953. Protein and amino acid requirements of the honeybee (Apis mellifica L.). - Physiol. Comp. Oecol. 3: $197-285$.

Hainsworth, F. R. and Wolf, L. L. 1976. Nectar characteristics and food selection by hummingbirds. - Oecologia 25: $101-$ 113.

Herrera, C. M. 1987. Components of pollinator "quality": comparative analysis of a diverse insect assemblage. - Oikos 50: 79-90.

Inouye, D. W. and Waller, G. D. 1984. Responses of honeybees (Apis mellifera) to amino acid solutions mimicking floral nectars. - Ecology 65: 618-625.

Jervis, M. A. and Boggs, C. L. 2005. Linking nectar amino acids to female butterflies. - Trends Ecol. Evol. 20: 585-587.

Keynan, S. and Kanner, B. I. 1988. Gamma-aminobutyric acid transport in reconstituted preparations from rat-braincoupled sodium and chloride fluxes. - Biochemistry 27: $12-17$.

Lanza, J, Smith, G. c., Sack, S. et al. 1995. Variation in nectar volume and composition of Impatiens capensis at the individual, plant, and population levels. - Oecologia 102: $113-119$.

Lord, E. M. 1982. Effect of daylength on open flower production in the cleistogamous species Lamium amplexicaule L. - Ann Bot 49: 261-263.

Mevi-Schutz, J. and Erhardt, A. 2003. Larval nutrition affects female nectar amino acid preference in the map butterfly (Araschnia levana). - Ecology 84: 2788-2794.

Mevi-Schutz, J. and Erhardt, A. 2004. Mating frequency influences nectar amino acid preference of Pieris napi. - Proc. R. Soc. Lond. B 271: 153-158.

Mevi-Schutz, J. and Erhardt, A. 2005. Amino acids in nectar enhance butterfly fecundity: a long awaited link. - Am. Nat. 165: 411-419.

Michener, C. D. 1979. Biogeography of the bees. - Ann. Miss. Bot. Gard. 66: 277-347.

Michener, C. D. 2000. The bees of the World. - The Johns Hopkins Univ. Press.

O'Brien, D. M., Fogel, M. L. and Boggs, C. L. 2002. Renewable and nonrenewable resources: amino acid turnover and allocation to reproduction in Lepidoptera. - Proc. Natl Acad. Sci. USA 99: 4413-4418.

O’Toole, C. and Raw, A. 1991. Bees of the world. - Blandford, London.

Pais, M.-S. S., Chaves, D. A. S., Neves, H. J. et al. 1986. Amino acid and sugar content of the nectar exudate from Limodorum abortivum (Orchidaceae). Comparison with Epipactis 
atropurpurea nectar composition. - Apidologie 17: 125136.

Percival, M. S. 1961. Types of nectar in angiosperms. - New Phytol. 60: 235-281.

Percival, M. S. 1965. Floral biology. - Pergamon.

Petanidou, T. 1991. Pollination ecology in a phryganic ecosystem. PhD thesis, (in Greek, with English summary). - Aristotelian Univ., Thessaloniki.

Petanidou, T. 2004. Temporal patterns of resource selection in plant-pollinator communities in the Mediterranean: what can they really tell us? - In: Arianoutsou, M. and Papanastasis, V. (eds), Ecology, conservation and management of Mediterranean climate ecosystems - Proc. 10th MEDECOS Conf., Rhodos, CD ISBN 9059660161. Millpress, Rotterdam.

Petanidou, T. 2005. Sugars in Mediterranean floral nectars: an ecological and evolutionary approach. - J. Chem. Ecol. 31: $1065-1088$.

Petanidou, T. and Ellis, W. N. 1993. Pollinating fauna of a phryganic ecosystem: composition and diversity. - Biodiv. Lett. 1: 9-22.

Petanidou, T. and Ellis, W. N. 1996. Interdependence of native bee faunas and floras in changing Mediterranean communities. - In: Matheson, A., Buchmann, S. L., O’Toole, C. et al. (eds), The conservation biology of bees. Linn. Soc. Symp. series No 18, Academic Press, pp. 201-226.

Petanidou, T. and Potts, S. G. 2006. Mutual use of resources in Mediterranean plant-pollinator communities: how specialised are pollination webs? - In: Waser, N. and Ollerton, J. (eds), Plant-pollinator interactions: from specialization to generalization. Univ. of Chicago Press, pp. 220-244.

Petanidou, T. and Smets, E. 1995. The potential of marginal lands for bees and apiculture: nectar secretion in Mediterranean shrublands. - Apidologie 26: 39-52.

Petanidou, T. and Smets, E. 1996. Does temperature stress induce nectar production in Mediterranean plants? - New Phytol. 133: 513-518.

Petanidou, T. and Vokou, D. 1993. Pollination ecology of Labiatae in a phryganic (East Mediterranean) ecosystem. - Am. J. Bot. 80: 892-899.

Petanidou, T., Ellis, W. N., Margaris, N. S. et al. 1995. Constraints on flowering phenology in a phryganic (East Mediterranean shrub) community. - Am. J. Bot. 82: 607620.

Petanidou, T., Goethals, V. and Smets, E. 1999. The effects of nutrient and water availability in the nectar production and nectary structure of the dominant Labiatae species of phrygana. - Syst. Geogr. Plant 68: 233-244.

Petanidou, T., Goethals, V. and Smets, E. 2000. Nectary structure of Labiatae in relation to their nectar secretion and characteristics in a Mediterranean shrub community does flowering time matter? - Plant Syst. Evol. 225: $103-$ 118.

Petanidou, T., Van Laere, A. J. and Smets, E. 1996. Change in floral nectar components from fresh to senescent flowers of Capparis spinosa L., a nocturnally flowering Mediterranean shrub. - Plant Syst. Evol. 199: 79-92.

Potts, S.G., Vulliamy, B., Roberts, S. et al. 2004. Nectar resource diversity organises flower-visitor community structure. - Entomol. Exp. Appl. 113: 103-107.

Pyke, G. H. and Waser, N. M. 1981. On the production of dilute nectars by hummingbird and honeyeater flowers. - Biotropica 13: $260-270$.

Romeis, J. and Wackers, F. L. 2000. Feeding responses by female Pieris brassicae butterflies to carbohydrates and amino acids. - Physiol. Entomol. 25: 247-253.

Roubik, D. W., Yanega, D., Aluja, M. S. et al. 1995. On optimal nectar foraging by some tropical bees (Hymenoptera: Apidae). - Apidologie 26: 197-211.

Soltis, D. E., Soltis, P. S., Endress, P. K. et al. 2005. Phylogeny and evolution of angiosperms. - Sinauer.

Southwick, E. E. 1982. Nectar biology and pollinator attraction in the north temperate climate. - In: Breed, M. C. D., Michener, C. and Evans, H. E. (eds), The biology of social insects. Westview Press, Boulder, pp. 19-23.

Stiles, F. G. and Freeman, C. E. 1993. Patterns in floral nectar characteristics of some bird-visited plant species from Costa Rica. - Biotropica 25: 191-205.

Thawley, A. R. 1969. The components of honey and their effects on its properties: a review. - Bee World 50: 51-60.

The International Plant Names Index. 2004. http://www. ipni.org.

Velleman, P. 2005. DataDesk version 6.2.1. - Data Description Inc., Ithaca.

Waser, N. and Ollerton, J. (eds) 2006. Plant-pollinator interactions: from specialization to generalization. - Univ. of Chicago Press.

Wolfersberger, M. G. 2000. Amino acid transport in insects. - Annu. Rev. Entomol. 45: 111-120.

Wykes, G. R. 1952. An investigation of the sugars present in the nectar of flowers of various species. - New Phytol. 51: 210215. 